\title{
Comments on Bette Furn's "Adjustment and the Near-Death Experience"
}

\author{
Joseph B. Geraci \\ University of Connecticut
}

Bette Furn's paper is a fine effort to assist people who appear to need adjustment. As a near-death experiencer (NDEr), however, I disagree with its basic question: What is it the NDEr is to adjust to, and why? The most difficult adjustment is being human again. The NDE is not a human experience; it does not involve variables. Instead of trying to make the NDEr conform to "here," why not spend that time and energy learning from the NDEr how to change "here" for the better? The experience does not happen to so many people only to have them come back and be reprogrammed into the same cultures and mistakes.

The NDEr is not a "new client population" for mental health practitioners. In fact, the situation is just the opposite: mental health practitioners, along with the rest of the world, are the clients of the NDEr. NDErs are trying to bring home to their "host" culture a very simple but important message. The "host" culture, however, has yet to listen or for that matter, to accept the messengers. Instead, the NDEr is subjected to mere curiosity by some and to dissection by others. If one truly wants to help NDErs, simply listen to them and share their love.

I apologize if I am being overly critical of Furn's paper. As an NDEr I become frustrated at being put in a category or labeled and put in a jar. I would much rather have people do things with me than to me.

There are only two kinds of people who can help an NDEr adjust to being human again: other NDErs, and those who have been touched by the love from the same source. All the intervention strategies and

Mr. Geraci is an administrator in the New Britain Public Schools and a Ph.D. candidate at the University of Connecticut. Requests for reprints should be sent to Mr. Geraci at 105 Ten Acre Road, New Britain, Ct 06052. 
counseling techniques in existence will fall short. I remember well my first encounter with other NDErs. I wondered what I would say and what they would say. I was nervous and even uncomfortable. When we met, however, it was one of the most beautiful feelings I have had since my experience. There was no need to say anything at all. Just looking into their eyes and sharing hugs was all that was needed; I could finally share my experience without saying a word. We spent most of our time talking about our families and work, just like "normal" people.

Every NDEr has returned for a very important reason; students of the many NDE case studies should know that by now. It is interesting to note that we were all taken from a certain place at a certain time, and returned to that same place and time, all with a common feeling of love, all with new lives. I would much rather see health professionals, clergy, and government officials provide a forum in which NDErs could share their knowledge. We are not in need of intervention or treatment. What we are in need of is to be listened to and given the opportunity to do the helping. Perhaps it's time to give the NDer a chance to "successfully intervene in the adjustment process" of life on this Earth. 